\title{
Narrativas autobiográficas sobre psoríase nas redes sociais: análise de depoimentos de usuários do YouTube
}

\author{
Autobiographical narratives about psoriasis on social media: analysis of testimonials from \\ YouTube users
}

Narrativas autobiográficas sobre la psoriasis en las redes sociales: análisis de testimonios de usuarios de YouTube

\section{Resumo}

A vivência de quem possui uma doença autoimune como a psoríase não só afeta a aparência física das pessoas, mas também seu estado psicossocial, podendo trazer importantes prejuízos à vida. Este estudo objetivou analisar a experiência de cronicidade, por psoríase, vivida e documentada na plataforma YouTube. Trata-se de um estudo de pesquisa documental, qualitativo, em que a coleta de dados foi através da seleção de vídeos da plataforma YouTube com narrativas autobiográficas sobre a psoríase. A coleta de dados ocorreu nos meses de fevereiro e março de 2021, recuperando seis vídeos disponíveis no YouTube. As transcrições dos vídeos foram submetidas à análise temáticoreflexiva e, posteriormente, discutidas a partir da literatura científica sobre a psoríase, sobretudo no campo da saúde mental. Foram produzidos dois temas principais: (1) descoberta e diagnóstico da psoríase; (2) sentimentos, preconceito em relação à psoríase e como isso afeta a qualidade de vida das pessoas acometidas pela doença. Os youtubers revelaram que a psoríase promoveu sentimentos negativos, com repercussões emocionais, físicas e sociais. As narrativas reforçaram a importância de um diagnóstico preciso e precoce para a melhor administração dos efeitos psicossociais dessa condição. Investigar de modo pormenorizado o papel dessa rede social na desconstrução de estigmas associados à psoríase é uma recomendação para novos estudos.

Palavras-chave: Psoríase; Estigma; Preconceito; YouTube.

\section{Abstract}

The experience of those who have an autoimmune disease such as psoriasis affects people's physical appearance and psychosocial status. It can bring significant harm to these people's lives. The study aimed to analyze the experience of chronic illness from psoriasis lived and documented on the YouTube platform. It is documental, qualitative research. The collected data was through the selection of videos from the YouTube platform with autobiographical narratives 
about psoriasis. Data collection took place in February and March 2021, retrieving six videos available on YouTube. The transcripts of the videos were subjected to thematic-reflective analysis and later discussed based on the scientific literature on psoriasis, especially in the field of mental health. Was produced two main themes: (1) psoriasis discovery and diagnosis; (2) feelings, prejudice towards psoriasis, and how it affects the quality of life of people affected by the disease. The YouTubers' narratives revealed that psoriasis promoted negative feelings, with emotional, physical, and social repercussions. The reports reinforced the importance of an accurate and early diagnosis for better management of the psychosocial effects of this condition. Investigating the role of this social network in the deconstruction of stigmas associated with psoriasis is a recommendation for further studies.

Keywords: Psoriasis; Stigma; Prejudice; YouTube.

\section{Resumen}

La experiencia de quienes tienen una enfermedad autoinmune como la psoriasis no solo afecta la apariencia física de las personas, sino también su estado psicosocial, puede traer daños significativos a la vida de estas personas. Este estudio tuvo como objetivo analizar la vivencia de la enfermedad crónica por psoriasis vivida y documentada en la plataforma de YouTube. Se trata de un estudio de investigación documental, cualitativo, en el que se recopilaron datos por medio de la selección de videos de la plataforma de YouTube con narrativas autobiográficas sobre la psoriasis. Se realizó la recolección de datos en febrero y marzo de 2021, recuperando seis videos disponibles en YouTube. Las transcripciones de los videos fueron sometidas a análisis temático-reflexivo y posteriormente discutidas en base a la literatura científica sobre psoriasis, especialmente en el campo de la salud mental. Se produjeron dos temas principales: (1) descubrimiento y diagnóstico de la psoriasis; (2) sentimientos, prejuicios hacia la psoriasis y cómo afecta la calidad de vida de las personas afectadas por la enfermedad. Los YouTubers revelaron que la psoriasis promovía sentimientos negativos, con repercusiones emocionales, físicas y sociales. Las narrativas reforzaron la importancia de un diagnóstico precoz y preciso para un mejor manejo de los efectos psicosociales de esta condición. Investigar en detalle el papel de esta red social en la deconstrucción de los estigmas asociados a la psoriasis es una recomendación para futuros estudios.

Palabras clave: Psoriasis; Estigma; Prejuicio; YouTube.

\section{Introdução}

A psoríase é uma doença de pele do tipo autoinflamatória, cíclica, não contagiosa e crônica. A etiologia está relacionada a fatores genéticos, ambientais e/ou de comportamento que causam uma alteração na função celular, gerando um quadro inflamatório. Esse quadro possui por características lesões com placas escamosas na pele sem local específico e de diferentes tamanhos. A doença pode ser classificada conforme as características das lesões, sendo: em placas ou vulgar (a mais comum); ungueal; do couro cabeludo; gutata; invertida; pustulosa e artropática (Sociedade Brasileira de Dermatologia [SBD], 2021).

Em um estudo realizado pelo núcleo de Ensino Superior em Ciências Humanas e da Saúde, aplicado em pacientes atendidos em clínica dermatológica, verificou-se 855 prontuários. Desse total, 32 (3,7\%) possuíam diagnóstico de psoríase. Destes, 17 (53,13\%) eram do sexo masculino, com idade média de 46 anos, e 15 (46,87\%) do sexo feminino. Uma das maiores comorbidades relacionadas à psoríase é a artrite psoriática, que atinge cerca de $20 \%$ a $30 \%$ dos pacientes com psoríase. Entretanto, nem todo paciente terá alguma queixa de artrite ou sintoma articular (Amarante et al., 2020).

É uma doença que afeta de $1 \%$ a 3\% da população no mundo. No Brasil, estudo aponta a prevalência de 0,92\% na região Norte e $1,88 \%$ no Sudeste. Não se trata de uma doença contagiosa, porém as lesões são dolorosas, a pele apresenta aspecto desfigurado, dependendo do grau da doença, gerando um grande impacto na qualidade de vida do paciente, ocasionando repercussões psicológicas, como danos na autoestima, na vida social e em termos da saúde mental (Pollo, 2020).

Dentre os impactos na qualidade de vida, a pessoa com psoríase pode apresentar alteração e/ou impedimentos na sua rotina diária. Além disso, a psoríase representa um grande estigma para esses pacientes. Em relação às repercussões no campo da saúde mental, esses pacientes podem vivenciar sentimentos de angústia, rejeição, vergonha, baixa autoestima, com autoimagem depreciativa, o que, inequivocamente, afeta a vida social e pessoal. Esse conjunto de sentimentos, a depender do estágio em que o paciente se encontra, pode estar associado à piora e ao aumento das lesões devido ao processo inflamatório e proliferação do aumento das células descamativas (Guerreiro et al., 2018).

Neste contexto, torna-se de grande relevância estudar a realidade de quem convive com a psoríase diariamente para fomentar o entendimento dos impactos nos diversos âmbitos da vida desses sujeitos, dimensionando de modo mais adequado a 
repercussão desses agravos na qualidade de vida. Diante disso, faz-se possível o desenvolvimento de ações/estratégias que possibilitem uma assistência mais adequada a essas pessoas justamente a partir da real identificação de suas necessidades de saúde e de cuidado.

As redes sociais têm sido cada vez mais acessadas na contemporaneidade, revelando um modo de interagir e se comunicar típico de nosso tempo histórico. Sua utilização perpassa pela incessante busca de informações, como fonte de estudo, curiosidades, desde momentos de entretenimento, lazer e distração. No mundo contemporâneo, as redes sociais permitem a interação entre as pessoas sem a necessidade do contato físico, o que pode aproximar pessoas que partilham determinados interesses e necessidades, como é o caso de pacientes com uma doença específica. Diante do adoecimento, portanto, as redes sociais podem funcionar como ferramentas de informação, de compartilhamento de dúvidas e experiências, bem como de construção de redes de apoio virtuais (Xavier et al., 2020). No entanto, nem sempre essas redes apresentam informações e orientações corretas do ponto de vista científico, o que também pode interferir na transmissão de práticas adequadas de saúde e de cuidado.

Uma das plataformas digitais mais acessadas no mundo é o YouTube, fundado por Chad Hurley, Steve Chen e Jawed Karim no ano de 2005. É uma plataforma na qual usuários enviam seus vídeos de diversos temas para alcançar o máximo de inscritos e visualizações. Os usuários produtores de conteúdo nesta plataforma são chamados de youtubers. Muitos transmitem em seus vídeos desde sua rotina de vida, descobertas de doenças, receitas, rotina de estudos, atividades de lazer, entre outros, o que torna a plataforma uma fonte rica e diversificada de conteúdo (Dure \& Ceolin, 2017).

No que se refere ao acesso a conteúdos no campo da saúde, estudos apontam para as potencialidades do emprego do YouTube. No entanto, é importante também assinalar que a plataforma pode ser um repositório de informações equivocadas (Villa et al., 2021), contribuindo para a divulgação de fake news e também de transmissão de orientações que podem pôr em risco a vida das pessoas. No campo da saúde, por exemplo, destaca-se como o movimento antivacina tem se fortalecido a partir dessa mesma plataforma (Costa, Viegas, Moreira, \& Abreu, 2020).

Apesar dessas considerações, deve-se destacar que o YouTube é a plataforma de vídeos mais acessada mundialmente e que ainda há uma escassa investigação científica sobre canais ou perfis voltados à divulgação de informações sobre a psoríase ou mesmo para compartilhamento de experiências de pessoas em tratamento. Diante desse panorama e considerando a necessidade de reconhecer, avaliar e validar iniciativas de informação em saúde que se sustentem em estudos científicos e em orientações oficiais, o objetivo deste estudo é analisar a experiência de cronicidade, por psoríase, vivida e documentada na plataforma YouTube.

\section{Metodologia}

Trata-se de uma pesquisa documental. Apesar de historicamente associados às pesquisas em ciências humanas e sociais, os estudos documentais ocupam um papel importante na construção de conhecimentos no campo das ciências da saúde, aspecto este que tem sido cada vez mais recuperado, sobretudo quando analisamos cenários complexos e mutáveis, como as redes sociais. Os estudos documentais, nesse sentido, permitiriam a construção de estudos descritivos com grande possibilidade de detalhamento de informações, bem como de acompanhamento ao longo do tempo, ampliando a compreensão de um dado fenômeno a partir de seus efeitos e transformações (Scorsolini-Comin, 2021). Embora não forneçam níveis robustos de evidências para a prática, podem disparar diferentes reflexões que sejam posteriormente investigadas a partir de diferentes métodos.

O corpus documental selecionado para a presente investigação é composto por vídeos publicados na plataforma YouTube, sendo, portanto, documentos em formato audiovisual. A coleta de dados ocorreu nos meses de fevereiro e março de 2021 utilizando-se da plataforma YouTube a partir do compartilhamento de vídeos enviados pelos usuários. Primeiramente foi 
feita a busca no campo de pesquisa na plataforma, utilizando filtros de busca com as palavras chave: psoríase, história com a psoríase e luta contra a psoríase. A partir dessa busca e de uma análise prévia dos registros, 10 vídeos foram selecionados para serem assistidos na íntegra. Após essa análise inicial foram incluídos no corpus analítico seis vídeos que relatavam a vivência com a psoríase e a descoberta da doença. Foram excluídos quatro vídeos que abordavam especificamente sobre tratamento e cura, não trazendo as experiências de usuários com psoríase. Posteriormente, os vídeos que compuseram o corpus foram assistidos minunciosamente e tiveram suas narrativas transcritas na íntegra e literalmente.

A transcrição do corpus foi submetida à análise temático-reflexiva de Braun e Clarke (2019), que consiste na produção de temas representativos das falas dos usuários. Não se trata de uma análise automatizada, mas justamente incorporando os efeitos de sentido em seus interlocutores. Participaram da construção e verificação dos temas duas pesquisadoras independentes. Possíveis desacordos nessa tematização foram discutidos com um terceiro pesquisador, todos com experiência na realização de estudos qualitativos.

Para organizar os temas foram elaboradas fichas. Para a sistematização do corpus foi utilizada a letra V (vídeo). Os vídeos foram ordenados de forma crescente utilizando numeração ordinal. Foi empregada a letra Y para se referir ao canal do usuário no YouTube, a fim de preservar a sua identidade.

A pesquisa foi realizada a partir de materiais de domínio público, não sendo necessária a aprovação e apreciação pelo Comitê de Ética em Pesquisa, haja vista que a Resolução nº 510/2016 do Conselho Nacional de Saúde, amparada pela Lei de Acesso à Informação ( $n^{\circ}$ 12.527/2011), dispensa pesquisas dependentes exclusivamente de informações de acesso público da submissão a um Comitê de Ética, desde que, conforme o Artigo 31, seja realizado o tratamento das informações pessoais de modo "[...] transparente e com respeito à intimidade, vida privada, honra e imagem das pessoas, bem como às liberdades $\mathrm{e}$ garantias individuais" (Brasil, 2011).

\section{Resultados e Discussão}

Para este estudo foram selecionados e analisados seis vídeos, compartilhados por cinco youtubers. Todos os vídeos selecionados e analisados para esta pesquisa referem-se à vivência com a doença psoríase, englobando todos os aspectos da vida (social, emocional, pessoal). O Quadro 1 apresenta a identificação dos vídeos que compuseram o corpus e características como número de visualizações, inscritos nos canais e curtidas (like).

Quadro 1: Relação de vídeos selecionados de acordo com o número decrescente de visualizações, do número de inscritos nos canais e curtidas.

\begin{tabular}{|c|c|c|c|c|}
\hline Código do Vídeo & $\begin{array}{c}\text { Canal do } \\
\text { Youtuber }\end{array}$ & $\begin{array}{c}\mathrm{N}^{\circ} \text { de } \\
\text { Visualizações }\end{array}$ & $\mathrm{N}^{\circ}$ de inscritos & $\begin{array}{c}\text { Curtidas } \\
\text { "Likes" }\end{array}$ \\
\hline V1 & Y1 & 226.766 & 547.000 & 11.000 \\
\hline V2 & Y2 & 24.852 & 7.820 & 1.400 \\
\hline V3 & Y3 & 1.410 & 10.400 & 59 \\
\hline V4 & Y4 & 742 & 235 & 45 \\
\hline V5 & Y5 & 281 & 1.200 & 55 \\
\hline V6 & Y5 & 217 & 1.200 & 39 \\
\hline
\end{tabular}

Fonte: Autores (2021).

Os vídeos com maiores visualizações foram V1 E V2, ao passo que os vídeos com menor visualização foram V5 e V6. Somando as quantidades de visualizações dos seis vídeos temos um total de 254.268 visualizações. Compreendemos, por esses quantitativos, que há uma grande procura em saber o que é essa doença, como ela afeta a vida das pessoas, tendo em vista que os comentários nos vídeos selecionados, em sua maioria, são de pessoas que já têm a doença ou a descobriram recentemente. 
Embora os vídeos V1 e V2 sejam o de maior repercussão, ou seja, os que têm mais visibilidade pelo público, vale destacar a relevância dos demais vídeos para o público que sofre com psoríase, haja vista que todos trazem informações pertinentes acerca da descoberta da doença e como cada usuário vivenciou esse processo, bem como os impactos em suas vidas.

O quantitativo de pessoas inscritas nos cinco canais do YouTube selecionados neste estudo foi de aproximadamente 567.855 mil inscritos. Observamos que a quantidade de inscritos em um canal favorece o aumento de visualizações entres os seguidores, impulsionando novos acessos.

Nesses vídeos os youtubers contam como foi descobrir a psoríase, os diagnósticos que muitas vezes eram equivocados, a vivência com a psoríase na vida pessoal, social, trabalho e sentimentos vividos em cada etapa da vida, além do preconceito que alguns sofriam. Em relação à comunicação verbal, alguns youtubers são bem alegres e comunicativos, outros demonstram sentimentos de tristeza, ficam muito emocionados ao lembrar de como seu corpo ficou com a doença e o preconceito que sofriam e sofrem até hoje.

Após a análise dos vídeos a partir das transcrições, dois temas foram produzidos: (1) a descoberta e diagnóstico da psoríase; (2) sentimentos, preconceito em relação a psoríase e como isso afeta a qualidade de vida das pessoas acometidas pela doença. A seguir esses temas serão melhor descritos e discutidos.

\subsection{A descoberta e diagnóstico da psoríase}

Este tema compete ao conteúdo dos vídeos analisados em que os youtubers pronunciam-se sobre como descobriram que tinham a doença e quais foram os sentimentos experienciados nesse momento. Nos vídeos V1, V2 e V3 os youtubers relatam que descobriram a psoríase na infância, como pode-se observar nas falas dos vídeos a seguir:

"[...] a primeira vez que tive contato com a doença eu tinha mais ou menos 12 anos, e ela se manifestou na minha pele [...] no meu caso eram bolinhas que descamam [...] eu sempre tinha na região da barriga [...]”. (V1/Y1)

"Eu tinha uma caspa severa na infância, minha mãe tratou essa caspa severa de todas as formas com receitinhas caseiras por isso nunca procurei tratamento [...]". (V2/Y2)

"[...] No meu caso é a gutata e eu tenho isso desde os oito anos [...]”. (V3/Y3)

Podemos observar que em dois relatos a psoríase teve início na infância dessas pessoas, de forma mais branda, o que dificultou o diagnóstico correto ou mais preciso. Dentre as classificações da psoríase, uma das consideradas mais raras em crianças é a vulgar, correspondendo a cerca de 4\% em relação a outras doenças de pele ocasionadas em menores de 16 anos. Esse tipo de psoríase tem uma alta incidência hereditária. Nos primeiros anos de infância, a manifestação mais comum da psoríase tende a ser o aparecimento de placas eritematosas, geralmente na região genital, glútea, sendo de tratamento difícil e persistente. Após surgem placas escamosas e eritematosas, em região do tronco e membros, e há também os que se manifestam-se no couro cabeludo (SBD, 2019).

Sabendo que a psoríase é uma das dermatoses pouco relatada em crianças, a sua prevalência nessa faixa etária ainda é desconhecida. Entretanto, entre 25-45 \% dos casos da doença tem incidência antes dos 16 anos. Com isso temos que $2 \%$ dos casos se dão até os dois anos de idade. Essa dermatose, quando tem seu surgimento no couro cabeludo, promove o aparecimento de escamas de coloração branca e espessa rente aos folículos pilosos, com leve vermelhidão sendo bem típico essas características (SBD, 2019).

Os relatos dos demais youtubers referem a descoberta da doença na fase adulta, e que o processo pelo qual passaram para ter um diagnóstico preciso da doença também foi repleto de adversidades e demandaram um tempo considerável. 
"[...] eu sempre era diagnosticada como pitiriase [...] em 2016 eu passei por um processo de separação, então provavelmente foi um momento mais estressante da minha vida [...] se antes aparecia em dois lugares agora apareceu em todo o corpo inteiro [...] essas bolinhas começaram a aumentar fui no médico que dizia que era pitiriase”. (V1/Y1)

Analisando a fala do youtuber Y1, podemos observar que a doença no início se manifestou de forma branda, apresentando lesões em alguns locais do corpo e de tamanho pequeno, mas como se trata de uma doença ligada ao emocional tende a se alastrar pelo corpo quando se passa por um momento estressante ou conturbado da vida. Podemos observar que se houvesse um tratamento adequado desde o início a doença não iria ter se alastrado por todo o corpo. Verificamos também que a psoríase é muito confundida com pitiriase, sendo de difícil diagnóstico médico e ocasionando um tratamento inadequado.

É de grande importância ter um diagnóstico preciso, sendo que em inúmeras vezes essa condição é avaliada de forma errônea, sendo confundida com outros distúrbios da pele que também causam lesões em formato de placas e que descamam. Conforme a doença tem sua evolução, estabelece-se um padrão descamativo característico da psoríase. Como normalmente é de fácil reconhecimento médico, não são necessários exames para diagnóstico. Todavia, é realizada a biopsia de pele para confirmação do diagnóstico (Moscardi \& Ogava, 2017).

"[...] a primeira vez que fui no dermatologista ele disse que era dermatite seborreica porque era no couro cabeludo [...] depois de um tempo fui diagnosticada com psoríase". (V2/Y2).

"[...] no final do ensino médio, me arrumando para o baile de formatura eu estava arrumando meu cabelo e aí na hora que a cabelereira pegou e falou tem um negócio na sua cabeça, parece impigem, na época não me dei conta [...]”. (V5/Y5)

"[...] no fim do ano tive uma caspa muito forte [...] fui num dermatologista e ele tratava como seborreia e disse que isso pode ser psoríase e tem a ver com o fundo emocional [...] dai outro médico me pediu biopsia, daí saiu o resultado ele olhou pra mim e disse é realmente psoríase doença que não tem cura, você vai conviver com ela pro resto da vida $[\ldots] ”(V 5 / Y 5)$

Os youtubers Y2 e Y5 relatam que nas consultas com o dermatologista o diagnóstico era dermatite seborreica, por conta de a psoríase ser localizada na região da cabeça e ter algumas características parecida com esse tipo de dermatite. Depois de anos convivendo com o tratamento errado, conseguiram ter acesso ao diagnóstico da psoríase.

Portanto, entende-se que a psoríase geralmente é confundida com outros tipos de doença dermatológicas que afetam tanto a autoestima como também causa prejuízos na qualidade de vida do indivíduo, por conta do tratamento errôneo. Por ser uma doença ainda pouco conhecida, ainda é muito confundida com outras doenças de pele mais comuns.

Geralmente na cabeça a doença tende a aparecer de forma leve, com pequenas placas, com escamas branco-prateadas (mais comum). Em torno de $80 \%$ das pessoas com psoríase apresentam lesões nessa região, sendo que um a cada quatro apresentam lesões exclusivamente no couro cabeludo (Bruni et al., 2021). Assemelha-se à caspa, pois pode causar prurido e após o paciente coçar, apresentar pele morta em flocos em seus cabelos ou em seus ombros (SBD, 2021).

“[...] passei em alguns dermatos, alergistas e nenhum conseguia um diagnóstico preciso [...] final de 2016 e início de 2017 comecei a parar de comer carne vermelha, a minha mãe disse que eu tinha alergia a carne e eu acreditei nisso [...] um clinico geral me indicou uma pomada e me indicou um dermatologista [...] passei por seis dermatologistas, três alergistas e dois clínicos, foi assim que descobri a psoríase [...]”. (V4/Y4) 
"[...] entre julho e agosto eu tive um surto dessas até então "manchinhas" explodiu no meu corpo inteiro, teve um dia que eu acordei estava sentindo uma coceira nas costas, quando olhei no espelho ele estava todo empelotado [...] ela parecia assim que estava descascando era vermelhinha pequenininha e aí ela ia crescendo e espalhando [...] fiquei três anos assim [...]”. (V4/Y4)

Nesses relatos é possível observar que os youtubers tiveram dificuldades em obter o diagnóstico preciso, após diversas tentativas para um tratamento eficiente. Isso mostra o quão importante é o estudo sobre essa doença para melhorar a qualidade de vida das pessoas acometidas por essa condição. Quanto mais cedo houver um tratamento adequado, mais rápida será a diminuição nas lesões.

Um diagnóstico correto primeiramente envolve um exame físico, observando as características das lesões na pele, como aspecto, localização e se há histórico familiar da doença. Não existem exames de sangue para a comprovação da psoríase. Essas lesões têm uma evolução habitual, tendo períodos em que há uma diminuição e também outros momentos da vida em que há um aumento significativo. Geralmente é uma doença assintomática, mas há casos em que os pacientes sentem prurido intenso. Podese também solicitar uma biopsia para comprovação da doença quando se há alguma dúvida no diagnóstico (Moscardi \& Ogava, 2017).

No começo da formação das placas da psoríase há a presença de edema na derme, há infiltramento perivascular constituídos por células T, $\mathrm{CD} 8$, monócitos e macrófagos. Subsequentemente, com aumento da espessidão do infiltrado aumentam e surgem os neutrófilos e as células CD 8 positivas, que passam a ser vistos na epiderme, gerando microabcessos de Munro, particularidades da psoríase (Conitec, 2021).

Um método utilizado para realizar a avaliação dos resultados terapêuticos e qualificar a psoríase a cada consulta chamase avaliação global pelo médico (Physician Global Assessment). O paciente obtém uma pontuação de 0-6 conforme a gravidade das lesões, sendo: 6-psoríase grave, 5-psoríase moderada-grave, 4-psoríase moderada, 3-psoríase leve moderada, 2-psoríase leve, 1- psoríase quase em remissão, 0- remissão completa. Há também um método de avaliação de índice de qualidade de vida dermatológico (DLQUI) que se refere a um questionário com 10 perguntas que analisam o impacto de doenças dermatológicas nos quesitos lazer estudo, relações pessoais e tratamento (Conitec, 2021).

Como a psoríase pode ser confundida com outras doenças, e ainda pode estar associada com outras patologias: cardiometabólicas, gastrointestinais, câncer e transtornos de humor, é necessário se atentar aos primeiros sinais e sintomas e buscar ajuda médica, preferencialmente, de um dermatologista. Quanto mais precoce o diagnóstico, mais efetivo será o tratamento, já que a psoríase, quando não tratada, impacta na qualidade de vida e dependendo do caso clínico, diminui a expectativa de vida (SBD, 2021).

\subsection{Sentimentos, preconceito em relação à psoríase e como isso afeta a qualidade de vida das pessoas acometidas pela doença}

Nos relatos dos youtubers podemos observar que a psoríase promoveu sentimentos totalmente diferentes em suas vidas. Esses sentimentos vão desde a aceitação até a rejeição da doença. Alguns vivenciaram o preconceito, despertando também a curiosidade das pessoas, sejam elas próximas ou não. Em função desse preconceito e do estigma produzido, algumas deixaram de realizar atividades de lazer e mudaram seus hábitos de vestimenta.

"[...] isso foi muito difícil pra mim e me afeta até hoje[...] ]estava afetando minha autoestima e eu acho que consegui lidar com isso de forma positiva porque eu não deixava de ir à praia, não deixava de ir a nada [...]”. (V1/Y1). 
"[...] até hoje tenho psoríase no ouvido, e descamavam muito e me deixava surda [...] o que aprendi com esse médico valeu pra vida toda porque eu aprendi que é preciso cuidar de toda minha saúde pra levar uma vida com psoríase numa boa [...]". (V2/Y2)

Analisando os vídeos dos youtubers Y1 e Y2 verificamos que eles tentaram lidar com a doença de forma positiva, levando em conta que a psoríase é uma doença crônica e que teriam que conviver com a mesma para o resto da vida. Mesmo que isso esteja afetando a autoestima, e a sua qualidade de vida, observamos que um dos youtubers aprendeu com um médico que precisava cuidar bem de sua saúde para ter uma vida tranquila com a psoríase, aceitando a doença.

Considerando que a psoríase é uma doença crônica, gera muitos prejuízos na qualidade de vida das pessoas por ela acometidas. Podem existir limitações nas atividades cotidianas, causando sentimento de vitalidade perdida. Por conta das lesões os indivíduos podem também se sentir rejeitados e envergonhados, ocasionando impactos negativos na autoestima e nas relações sociais (Guerreiro et al., 2018).

Em relação ao cotidiano há muitas incertezas para quem vive com uma doença crônica: as pessoas lidam com um novo jeito de ver a vida, mudam a sua rotina, seus hábitos, podendo sofrer com o processo de adaptação. Devido a isso o diagnóstico é cercado por sentimentos desde a tristeza, angústia e contestação (Guerreiro et al., 2018).

"[...] dai saiu o resultado da biopsia ele olhou pra mim e disse é realmente psoríase doença que não tem cura você vai conviver com ela pro resto da vida [...] naquele momento eu procura chão, eu procurava força, eu lembro nítido o pavor que eu sentia". (V5/Y5)

"[...] sofri bullying no colégio por causa disso [...] me chamavam de dálmata vê se pode uma coisa dessa [...] verão inteiro andei de calça comprida por medo por vergonha [...] eu sofri muito chegava em casa chorando [...]”. (V3/Y3)

Nesses relatos podemos observar a repercussão psicossocial da psoríase. Os sentimentos podem envolver descrença e inconformismo, gerando sofrimento emocional. Há também, muitas vezes, o sentimento de vergonha pela aparência, podendo mudar o estilo de se vestir para que não mostrem tanto as lesões, tentando de uma forma ou outra escondê-las. Isso reforça os achados do estudo de Silva e Faro (2019) realizado com 72 pacientes adultos, com psoríase, atendidos no Ambulatório de Dermatologia do Hospital Universitário da Universidade Federal de Sergipe, de que 48,6\% dos participantes (pacientes com psoríase) apresentaram sintomas depressivos. Este achado nos leva à necessidade de refletir sobre o modo como a saúde mental desses pacientes tem sido pensada pelos equipamentos de cuidado e dentro dos tratamentos tradicionais para essa condição crônica.

Estudo realizado em Portugal apresentou que as variáveis preditoras para o surgimento de sintomas depressivos, de ansiedade e estresse foram o autojulgamento, o evitamento experiencial/inflexibilidade psicológica, os anos de escolaridade e a vergonha associada à psoríase. Constatou-se que as áreas da vida mais afetadas em decorrência da doença são as relações íntimas, as atividades de lazer e o estudo/trabalho (Mendes, 2019).

Em outro estudo, um grupo de pessoas diagnosticadas com psoríase, comparado ao grupo controle, apresentou mais falta de clareza em suas próprias emoções, dificuldades na autoaceitação e controle de impulsos frente a emoções negativas. Ainda nesse grupo, foram evidenciadas maiores taxas de alexitimia grave, depressão, ansiedade e saúde mental debilitada (Innamorati et al., 2016). Assim, a literatura científica parece referir, de modo evidente, que o estresse pode desencadear ou piorar a doença, comumente quando associado com outros sintomas, como os de ansiedade e de sobrecarga emocional (Rua et al., 2021). Nesse sentido, a melhora na autoestima e bem-estar de pessoas com doenças cutâneas contribui para um tratamento efetivo (Isidorio et al., 2020). 
Pessoas com psoríase possuem mais chances de terem sintomas depressivos devido ao comprometimento psicoemocional, sendo necessário acompanhamento psicológico para promoção da saúde, qualidade de vida e melhora no enfrentamento da doença. Além disso, a associação entre psoríase e sintomas depressivos elevam o sofrimento ocasionado por essa doença de pele (Silva \& Faro, 2019).

A exacerbação das lesões em todo o corpo faz parte da forma mais grave da psoríase, causando limitações e desfigurações físicas, favorecendo a discriminação, o preconceito e a produção do estigma. Essas lesões podem ocasionar prejuízos psicossociais, promovendo sintomas como depressão e ansiedade, conforme relatos a seguir:

"Eu sofri bastante preconceito, quando eu estava no auge da crise, as pessoas tinham nojo, as pessoas achavam que era uma doença contagiosa, sendo que não é psoríase não passa de uma pessoa para outra [...]". (V1/Y1)

"As pessoas ficavam olhando, perguntavam, queriam tocar etc... felizmente a maiorias das pessoas que agiam assim eram mais distantes de mim [...]". (V1/Y1)

"Foi difícil pra mim entender o meи corpo, o que estava acontecendo e de aceitar que eu estava com aquelas manchas no meu corpo que eu ia sair na rua daquela forma e que eu ia encarar as pessoas me olhando [...]” (V1/Y1)

O youtuber 1 relata o preconceito vivenciado, destacando que as pessoas não entendiam do que se tratavam aquelas lesões e então o questionavam se doíam, quais eram os sintomas, se era contagiosa como outros tipos de doença de pele, entre outras abordagens promotoras de desconforto emocional. Devido a esses tipos de questionamento a pessoa pode evitar frequentar locais públicos, mudando toda a sua rotina e buscando a sua preservação. Contudo, esse movimento de isolamento do mundo social pode prejudicar muito a vida de quem convive com a psoríase.

Mesmo sabendo que não se trata de uma doença contagiosa, por exemplo, as pessoas com psoríase tendem a se preocupar com esse aspecto, levando em conta o receio das pessoas ao seu redor (Guerreiro et al., 2018). Esse é apenas um dos movimentos que podem ocorrer na experiência de uma pessoa com psoríase. Assim, é de suma importância o desenvolvimento de ações educativas em saúde a fim de esclarecer a população, minimizando o sofrimento emocional desses pacientes, bem como a oferta de apoio psicológico a esse público em específico (Guedes \& Viana, 2020).

Como já mencionado, o estresse é um fator primordial para o desenvolvimento e agravamento da psoríase, podendo também atuar no desencadeamento e piora de quadros psiconeurológicos. Portanto, dentre as condições que estão relativamente associadas à psoríase temos a ansiedade, o abuso de substâncias, a depressão e as disfunções sexuais. A somatização desses aspectos afeta os pacientes promovendo pensamentos relacionados à morte, isolamento social, perda ou alteração de emprego, aumento na chance de divórcio e predisposição a comportamentos autodestrutivos devido à sua gravidade e o impacto biopsicossocial (Rodrigues et al., 2020).

"Em 2005 eu fui em uma entrevista e ficava pensando em como falar que tinha psoríase [...] nessa época eu usava blusa 3/4, saia midi [...]". (V5/Y5)

"Uma vez eu estava no serviço e o ar condicionado não estava funcionando daí eu prendi meu cabelo e como eu tinha muitas lesões aqui atrás, um senhor passou atrás de mim, e disse: eu vou the dizer isso que você tem, você vai lá na farmácia de veterinário e compra um sabonete para sarna e você passa que vai ficar boazinha [...]". (V6/Y5)

"Ali naquele momento o homem me prescrevendo remédio para sarna [...] eu comecei a ficar depressiva, eu não tinha mais vontade de fazer mais nada eu não tinha mais saco pra explicar que aquilo não era sarna e não é contagioso $[\ldots] " .(V 6 / Y 5)$ 
Constatamos nesses relatos o quanto a psoríase pode afetar e modificar toda uma vida, trazendo prejuízos como um todo. Na fala do Y5 podemos constatar que para não sofrer especulações em torno de suas lesões, e como maneira de evitar olhares preconceituoso ou até mesmo curiosidades das pessoas, optou por vestir-se de forma que não mostrasse tanto suas lesões. Já no caso do Y5/V6, verificamos o quanto é desgastante para a pessoa com a doença explicar reiteradas vezes o que é essa condição e de que se trata de um quadro autoimune e não contagioso. Ainda em relação a este último relato, nota-se um grande estigma em relação à doença, associando-a a outras condições em caráter pejorativo, o que afeta a saúde mental não apenas por essa exposição, mas pelo modo como tais estigmas acabam sendo reforçados ao longo do tempo e a partir de diferentes interações.

Em estudo realizado com pacientes de um ambulatório, evidenciou-se que a psoríase influenciou ou impossibilitou a atividade profissional devido à gravidade da doença. A perceptibilidade das lesões é um dos fatores apontado pelo afastamento do trabalho. Com a psoríase os participantes também relataram que o isolamento social passou a ser parte do seu cotidiano, abdicando de atividades de recreação como ir à praia, festas, academia, entre outros (Guerreiro et al., 2018).

A partir do diálogo com a literatura científica e tendo como referências os relatos desses youtubers, compreende-se que as redes sociais, como o YouTube, podem desempenhar um papel importante no sentido de justamente expor essas pessoas, em um processo de recusa ao isolamento. A exposição dessas pessoas e de suas histórias pode promover um movimento importante não apenas no sentido desses pacientes assumirem sua condição, mas de promoverem movimentos de inclusão e de acolhimento justamente por uma compreensão sobre a psoríase em suas vidas. Assim, os relatos aqui recuperados não possuem a função de legitimar determinadas informações sobre a psoríase, mas permitir o compartilhamento dessas experiências. Acessar essas redes e entrar em contato com essas narrativas autobiográficas em relação ao adoecer pode ser uma forma de promover a aproximação, o acolhimento e também a ressignificação de estigmas, funcionamento, em certa medida, como estratégia de promoção de saúde mental, o que pode ser melhor endereçado em estudos vindouros e a partir de diferentes delineamentos metodológicos para a investigação das redes sociais.

\section{Considerações Finais}

A partir da análise dos dados obtidos pelas narrativas dos youtubers, observamos que há uma grande recorrência de diagnósticos equivocados e tardios, resultando em tratamentos inadequados e causando maiores impactos na qualidade de vida da pessoa acometida pela psoríase. Uma vez que o diagnóstico da psoríase é basicamente clínico, é imprescindível que os profissionais de saúde tenham conhecimento sobre essa patologia, a fim de identificar suas características de modo que se tenha diagnósticos precisos e tratamento adequado.

Considerando que a psoríase causa impacto negativo tanto fisicamente como afeta outros aspectos da vida pessoal, social, emocional e profissional, os profissionais devem assistir às pessoas acometidas por essa patologia não apenas visando ao tratamento clínico para o controle das lesões, mas oferecendo ferramentas que auxiliem na mitigação do sofrimento psíquico causado pela doença. Esse impacto negativo na qualidade de vida do paciente pode desencadear doenças como ansiedade e depressão, por conta de sofrerem discriminação e estigmatização. Por isso, faz-se necessário o estabelecimento de diversas estratégias para promoção da saúde mental e prevenção de agravos, bem como a importância de se realizar ações para melhor esclarecimento da população, desmistificando a doença e minimizando o sofrimento emocional desses pacientes. A veiculação dessas narrativas autobiográficas, como as compartilhadas pelo YouTube e recuperadas no presente estudo, pode ser um caminho importante nesse sentido, o que deve continuar fomentando outras investigações.

Para além dessas considerações específicas em relação às potencialidades das redes sociais na informação sobre essa condição, no decorrer do estudo observamos que não há muitos estudos sobre a psoríase no Brasil, sendo recomendado novas investigações acerca da temática para que possamos ter dados mais precisos sobre a prevalência desta doença no país, assim como sobre os impactos originados pela mesma. De igual monta, favorecer o compartilhamento de experiências sobre o adoecer 
crônico, como as relacionadas à psoríase, pode ser uma forma não apenas de enfrentamento do ponto de vista psíquico, mas também uma estratégia de formação e de informação em saúde, de promoção de cuidado e desconstrução de estigmas.

\section{Referências}

Amarante, M. S. D. L. D. et al. (2020). Perfil epidemiológico dos pacientes com diagnóstico de psoríase atendidos na BWS, BWS Journal, 3 , e20080094.

Braun, V., \& Clarke, V. (2019). Reflecting on reflexive thematic analysis. Qualitiative Research in Sport, 11(1), 1-9. https://doi.org/10.1080/2159676X.2019.1628806

Bruni, F. et al. (2021). Clinical and trichoscopic features in various forms of scalp psoriasis. Journal of the European Academy of Dermatology and Venereology, 35(9), 1830-1837. https://doi.org/10.1111/jdv.17354

Conitec (2021). Protocolo Clínico e Diretrizes Terapêuticas da Psoríase. Autor. http://conitec.gov.br/images/Consultas/Relatorios/20 21/20210615_Relatorio_Psoriase_CP_52.pdf

Costa, B. B., Viegas, D. J., Moreira, T. A., \& Abreu, P. A. (2020). O movimento antivacina no YouTube nos tempos de pós-verdade: Educação em saúde ou desinformação? Revista Mídia e Cotidiano, 14(1), 220-239. https://doi.org/10.22409/rmc.v14i1.38210

Dure, D. M., \& Ceolin, P. (2017). O crescimento do youtube no Brasil e a popularidade do canal nostalgia. https://www.riobrancofac.edu.br/site/doc/simposios/2016/O-crescimento-do-youtube-no-Brasil_Deborah-Dure.pdf

Brasil (2011). Presidência da República da Casa Civil. Subchefia para Assuntos Jurídicos. Lei $n^{o} 12.527$, de 18 de novembro de 2011. Autor.

Guerreiro, T. N. et al. (2018). Alterações no cotidiano de pessoas acometidas por psoríase. Revista Enfermagem UERJ, 26, e28332. https://doi.org/10.12957/reuerj.2018.28332

Guedes, D. R., \& Viana, E. S. (2020). Psoríase e sua ligação com os aspectos psicológicos. Revista PubSaúde, 3, a047. https://dx.doi.org/10.31533/pubsaude3.a047

Innamorati, M. et al. (2016). Health-related quality of life and its association with alexithymia and difficulties in emotion regulation in patients with psoriasis. Comprehensive Psychiatry, 70, 200-208. https://doi.org/10.1016/j.comppsych.2016.08.001

Isidorio, E. C. et al. (2020). O uso do tratamento psicológico na melhora da qualidade de vida de pacientes dermatológicos: uma revisão integrativa da literatura. Research, Society and Development, 9(10), e7699109191. https://doi.org/10.33448/rsd-v9i10.9191

Mendes, R. C. A. (2019). Processos de regulação emocional em pessoas com o diagnóstico de Psoríase. (Dissertação) - Escola Superior de Altos Estudos, Instituto Superior Miguel Torga.

Moscardi, E. R., \& Ogava, S. E. M. (2017). Psoríase: etiologia, diagnóstico e tratamento. Revista Uningá Review, $29(2), 70-74$.

Pollo, C. F. (2020). Fatores associados à qualidade de vida em pacientes com psoríase: um estudo transversal. (Tese) - Universidade Estadual Paulista.

Rodrigues, J. M. C. et al. (2020). Estresse e psoríase: novas abordagens no tratamento. Revista Eletrônica Acervo Científico, 12, e4638. https://doi.org/10.25248/reac.e4638.2020

Rua, M. O. et al. (2021). Influências da depressão na psoríase: uma relação bidirecional. Revista Eletrônica Acervo Científico, 23, e5650. https://doi.org/10.25248/reac.e5650.2021

Scorsolini-Comin, F. (2021). Projeto de pesquisa em ciências da saúde: guia prático para estudantes. Vozes.

Sociedade Brasileira de Dermatologia [SBD] (2019). Dermatologistas comemoram publicação pelo Governo de parâmetros mínimos para o atendimento de pacientes com psoríase. https://www.sbd.org.br/noticias/conquista-da-sbd-dermatologistas-comemoram-publicacao-pelo-governo-de-parametros-minimospara-o-atendimento-de-pacientes-com-psoriase/.

Sociedade Brasileira de Dermatologia [SBD] (2021). Psoríase. https://www.sbd.org.br/dermatologia/pele/doencas-e-problemas/psoriase/18/.

Silva, B. F. P., \& Faro, A. (2019). Regulação emocional e sintomas depressivos em pacientes com psoríase. Revista de Psicología (Santiago), 28(2), 1-10. https://dx.doi.org/10.5354/0719-0581.2020.55656

Villa, L. S. C., Mello, A. D. C., Gonçalves, J. V., Silva, T. M. G., \& Bernuci, M. P. (2021). Avaliação da qualidade dos vídeos sobre câncer de mama mais visualizados no YouTube: relevância para promoção da saúde da mulher. Reciis - Revista Eletrônica de Comunicação, Informação \& Inovação em Saúde, 15(3), 648-664. https://doi.org/10.29397/reciis.v15i3.2342

Xavier, F. et al. (2020). Análise de redes sociais como estratégia de apoio à vigilância em saúde durante a Covid-19. Estudos Avançados, 34(99), 261-282. https://doi.org/10.1590/s0103-4014.2020.3499.016 